\title{
Archipel
}

ARCHIPEL Études interdisciplinaires sur le monde insulindien

$94 \mid 2017$

Varia

\section{Pulo Ka Satu. Warisan Sejarah Awal Persuratan Melayu Pulau Pinang (Pulo Ka Satu. Patrimoine de l'histoire des débuts de la littérature malaise de Penang)}

Monique Zaini-Lajoubert

\section{(2) OpenEdition}

Journals

Electronic version

URL: http://journals.openedition.org/archipel/468

DOI: $10.4000 /$ archipel.468

ISSN: 2104-3655

\section{Publisher}

Association Archipel

Printed version

Date of publication: 6 December 2017

Number of pages: 233-242

ISBN: 978-2-910513-78-8

ISSN: 0044-8613

\section{Electronic reference}

Monique Zaini-Lajoubert, «Pulo Ka Satu. Warisan Sejarah Awal Persuratan Melayu Pulau Pinang (Pulo Ka Satu. Patrimoine de l'histoire des débuts de la littérature malaise de Penang) », Archipel [Online], 94 | 2017, Online since 06 December 2017, connection on 25 September 2020. URL : http:// journals.openedition.org/archipel/468; DOI : https://doi.org/10.4000/archipel.468 
Jelani Harun, Pulo Ka Satu. Warisan Sejarah Awal Persuratan Melayu Pulau Pinang (Pulo Ka Satu. Patrimoine de l'histoire des débuts de la littérature malaise de Penang), Kuala Lumpur, Perpustakaan Negara Malaysia (Bibliothèque nationale de Malaisie), 2014, 314 p. (bibliographie, index, illustrations). ISBN 978-967-931-255-3.

L'auteur de cet ouvrage, Jelani Harun, est un spécialiste malaisien des manuscrits malais, ayant publié, entre autres, un certain nombre d'ouvrages, comme son édition critique d'une partie du Bustan al-Salatin (Le jardin des rois) de Nuruddin Ar-Raniri $(2004 \text { et } 2008)^{3}$, et son analyse de cette même œuvre $(2009)^{4}$, ou celle, comparative, d'undang-undang (lois) (2008) .

Dans l'ouvrage dont nous donnons ici le compte rendu, l'auteur se penche sur les activités intellectuelles en malais d'une région particulière de Malaisie, Penang, depuis ses origines jusqu'au début du $\mathrm{XX}^{\mathrm{e}}$ siècle. Dans le présent ouvrage, qu'il dédie à tous les auteurs et copistes de manuscrits malais ${ }^{6}$, Jelani Harun étudie les textes en malais produits par divers groupes ethniques de Penang (Melayu jati [Malais de souche]), Jawi Peranakan [Indiens musulmans nés en Malaisie], Darah Keturunan Keling [descendants d'Indiens du sud], Darah Keturunan Arab [descendants d'Arabes], etc.), ainsi que par Francis Light, à l'origine de la colonie britannique de Penang. Les textes en question ne sont pas uniquement des manuscrits en jawi (malais en caractères arabes). Ils sont aussi imprimés, pour la plupart également en jawi, et même oraux. Ils sont de natures diverses (historiques, appartenant aux belles-lettres, traités religieux, lettres, accords, chansons, etc.). L'auteur prend donc le mot persuratan « littérature » de son titre dans un sens plus large que celui de belles-lettres en incluant toute production (écrite ou orale) en malais. Il agrémente son ouvrage d'un assez grand nombre d'illustrations (cartes, listes, photos (pour la plupart de manuscrits, de livres et de périodiques), etc.), de même que d'extraits de textes ou, plus rarement, de textes complets, étudiés dans son ouvrage.

Il divise son étude en cinq parties. Dans la première, il examine des documents, aussi bien anglais, chinois que malais, de tous genres (récits de voyage, mémoires, notes, romans, études, etc.) sur Penang, permettant d'éclairer l'histoire ancienne de l'île. Il explique que c'est dans les années 2000 que les recherches sur celle-ci se sont intensifiées, sous l'impulsion d'universitaires, en particulier de l'Universiti Sains Malaysia (USM), constatant que le processus de développement de Penang délaissait de plus en plus ses aspects historique et culturel.

3. Jelani Harun, éd., Bustan al-Salatin (Bab Pertama dan Kedua) Karangan Nuruddin Ar-Raniri (Le jardin des rois (premier et second chapitres), de Nuruddin Ar-Raniri), Kuala Lumpur, Dewan Bahasa dan Pustaka (Siri Warisan Sastera Klasik) (série Patrimoine de la Littérature classique), 2004, 443 p. ; Jelani Harun, éd., Bustan al-Salatin (Bab Ketiga), Kisah Raja-Raja yang Adil, Nuruddin Ar-Raniri (Le jardin des rois (troisième chapitre), Histoire des rois justes, de Nuruddin Ar-Raniri), Kuala Lumpur, Dewan Bahasa dan Pustaka (Siri Warisan Sastera Klasik), 2008, $349 \mathrm{p}$.

4. Jelani Harun, Bustan al-Salatin (The Garden of Kings), A Malay Mirror for Rulers, Pulau Pinang, Penerbit Universiti Sains Malaysia, 2009, 352 p.

5. Jelani Harun, Undang-Undang Kesultanan Melayu dalam Perbandingan (Les lois des sultanats malais, une comparaison), Pulau Pinang, Penerbit Universiti Sains Malaysia, 2008, 454 p. 6. «Memperingati semua pengarang dan penyalin manuskrip Melayu». 
L'auteur donne, entre autres, des indications sur le nom de l'île, mentionnée pour la première fois sur la fameuse carte de navigation dite de l'amiral Zheng $\mathrm{He}$ (XV $\mathrm{XV}^{\mathrm{e}}$ siècle), sous l'appellation de Bin Lang Yu ou Pin Lang hsu. Aux XVI et XVII ${ }^{\mathrm{e}}$ siècles, des voyageurs européens l'ont appelée Pulo Pinaom, Pulo Pinaon, Pulo Panying ou Pulo Pinang. Elle était donc connue bien avant que Francis Light n'en fasse une colonie anglaise en 1786. Les Malais ont, de leur côté, une tradition orale dans laquelle l'île est appelée Pulo Ka Satu, nom que l'auteur indique dans le titre de son ouvrage. Celleci concerne Nakhoda (Capitaine) Ragam, un personnage légendaire présent dans la tradition orale de Penang, mais aussi de Perak, de Johor, de Malacca et de Brunei Darussalam. On ne sait pas qui était en réalité Nakhoda Ragam. L'une des hypothèses avancée est qu'il s'agirait du Sultan Bolkiah de Brunei qui portait justement le surnom de Nakhoda Ragam. Il aurait vogué jusqu'à Penang à la fin du XV ou au début du $\mathrm{XVI}^{\mathrm{e}}$ siècle et aurait donné à cette île le nom de Pulo Ka Satu.

Quant au nom de Pulau Pinang «L'île de l'aréquier» (Areca catechu), sous lequel on la connaît aujourd'hui, il est apparu après qu'y soit plantée cette espèce, bien que l'on ne sache pas très bien dans quelles conditions. Selon Jelani Harun (p. 27), ce toponyme est venu sur le devant de la scène quand le sultan de Kedah, qui avait alors Penang sous son autorité, menacé par le Siam et la Birmanie, signa, dés 1771, avec la Compagnie anglaise des Indes orientales, plusieurs accords commerciaux. Il ajoute que « Le summum de ces accords fut finalement atteint le 11 août 1786 quand Francis Light réussit à obtenir l'autorisation du Sultan Abdullah Mukarram Shah (1778-1798) d'occuper Penang ${ }^{7} »$.

La signature d'accords en 1771 peut paraître surprenante. Plusieurs études parlent d'une forme de coopération entre la Compagnie et le sultan de Kedah, Muhammad Jiwa (1710-1778), la première cherchant à obtenir certains avantages facilitant ses activités commerciales, le second espérant une aide militaire contre ses ennemis, comme les Bugis de Selangor et de Riau ainsi que le Siam. Il est question, par exemple, de « l'association éphémère entre Kedah et la Compagnie anglaise des Indes orientales en 1770-71 $»^{8}$, le sultan ayant « promis la cession de la côte près de Kuala Kedah en échange d'une aide lors d'une attaque contre Selangor " ${ }^{9}$. On explique aussi qu'une attaque de « mercenaires bugis » (Bugis mercenaries) en 1771 à Kedah, « mena à un renforcement des relations avec les Anglais dont le sultan recherchait l'aide $\gg^{10}$. Des accords à cette fin entre les deux parties cette année-là sont rarement évoqués. C'est cependant le cas de Mahani Musa qui fait état d'un accord signé en avril 1771, «considéré comme étant le premier traité de défense signé entre un État

7. « Kemuncak perjanjian akhirnya terlaksana pada 11 Ogos 1786 apabila Francis Light berjaya mendapatkan kebenaran Sultan Abdullah Mukarram Shah (1778-1798) untuk menduduki Pulau Pinang ».

8. " the short-lived association between Kedah and the EIC in 1770-1 " (Barbara Watson Andaya et Leonard Y. Andaya, A History of Malaysia, Houndmills, Basingstoke, Hampshire, et Londres, The Macmillan Press, 1982, p. 103).

9. "promised the cession of the coast near Kuala Kedah in return for support in an attack on Selangor », ibid.

10. «led to an increase in relations with the British from whom the Sultan sought assistance. » (Rodolphe de Koninck, "Alor Setar, the Capital of Kedah : a City to Govern Agriculture », Archipel 36, 1988. Villes d'Insulinde (I), p. 149 et 150). 
malais et les Anglais $\rangle^{11}$. Phillip E. Jones indique, pour sa part, qu'une rencontre entre Francis Light et le sultan Muhammad Jiwa à Kedah a eu lieu en avril 1771, pour lui demander l'autorisation d'y établir un comptoir. Le sultan accepta, à condition que les Anglais lui garantissent une protection contre le Siam, ce qu'ils refusèrent ${ }^{12}$.

La deuxième partie est consacrée aux manuscrits malais en jawi originaires de Penang. Ces derniers, en majorité des copies, les œuvres de création étant rares, bien souvent, ne comportent ni date, ni nom d'auteur, de copiste et même de nom de lieu. À Penang, qui n'avait pas à sa tête de sultan, la production des manuscrits ne se faisait pas dans le palais du souverain ou chez des notables, mais chez des particuliers ou dans les bureaux de la Compagnie. Il n'existe pas de catalogue des manuscrits de Penang. C'est donc en consultant les catalogues des manuscrits des bibliothèques de Malaisie et de l'étranger, et en allant examiner les manuscrits sur place, aussi bien dans son pays, qu'en Grande-Bretagne, en France, ou encore aux Pays-Bas, que Jelani Harun a pu en identifier comme ayant été produits à Penang.

Les plus anciens sont, selon lui, les lettres de Francis Light, rédigées entre 1786 et 1794, échangées la plupart du temps avec des souverains et des hauts personnages malais. La School of Oriental and African Studies (SOAS) de Londres possède la plus grande collection de ces lettres. Il en existe aussi en France. La Bibliothèque nationale à Paris en compte une centaine, qui seraient des copies des lettres de Londres réalisées par Édouard Dulaurier (1807-1881), le premier enseignant de malais de l'École des Langues orientales vivantes. La Bibliothèque municipale de Tournus en a trente-huit, que l'auteur étudie plus particulièrement dans un chapitre de cette partie ${ }^{13}$. Il s'agirait de copies de certaines copies de Paris ou de copies d'originaux de Londres, réalisées par un copiste inconnu.

L'auteur consacre un chapitre à un accord de 1791 entre Francis Light et Sayid Hussain Aidid d'Aceh. Y sont fixées les conditions de résidence à Penang de ce dernier et de son groupe. Il termine cette deuxième partie par l'analyse de trois copies, provenant de Penang, de grandes œuvres malaises (Karya Agung Melayu), à savoir Hikayat Hang Tuah (Histoire de Hang Tuah), Hikayat Merong Mahawangsa (Histoire

11. " considered to be the first defensive agreement ever signed between a Malay state and the British » (« Early History: Penang Before 1786 », in Muhammad Haji Salleh, éd. (traduit par Leelany Ayob et Ng Wai Queen), Early History of Penang (USM, 2012, EPUB, 2015), https://books.google.fr/books?id=gPekCgAAQBAJ\&pg=PT4\&lpg=PT4\&dq=Early+History + of + Penang $+(U S M,+2012,++$ EPUB,+2015$) \&$ source=bl\&ots=KF8-3-IyuN\&sig=Ujm8tbUuRExYdAjZ2HbSkW7MIKU\&hl=fr\&sa=X\&ved=0ahUKEwiP_O7dhaTVAhXGHxoKHaa\#v=onepage\&q=Early $\% 20$ History $\% 20$ of $\% 20$ Penang $\% 20$ (USM $\% 2$ C $\% \overline{2} 02012 \% 2 \mathrm{C} \% 20 \% 20$ EPUB $\% 2 \mathrm{C} \% 20$ 2015)\& $\mathrm{f}=$ false).

12. Cf. Mariners, Merchants \& The Military too. A History of the British Empire, P J Publishing, 2011,https://books.google.fr/books?id=2v-UAwAAQBAJ\&pg=PT155\&lpg=PT155\&dq=treaty+between + the + british + india + company + and + the + sultan + of + kedah + in $+1771 \&$ source $=$ bl\&ots $=P-$ DawSyGwqZ\&sig=Gjx9 wxBRh4RWZu-jwl5ykNrvNk\&hl=fr\&sa=X\&ved=0ahUKEwiLjaiR2KbVAhUKBBoKHbhfB28Q 6 AEIaDAI\#v=onepage \&q=treaty $\% 20$ between $\% 20$ the $\% 20$ british $\% 20$ india $\% 20$ company $\% 20$ and $\% 20$ the $\% 20$ sultan $\% 20$ of $\% 20$ kedah $\% 20$ in $\% 201771 \& \mathrm{f}=$ false).

13. Cf. l'article de Jelani Harun, "À la recherche de manuscrits malais en France, avec une mention toute particulière sur les lettres de Francis Light », paru dans Archipel 89 (2015, p. 3961), que nous avons adapté d'une communication en malaisien de l'auteur intitulée « Menjejaki Manuskrip Melayu dan Surat-Surat Francis Light di Perancis » (Perpustakaan Negara Malaysia, 6 mai 2014. http//myrepositori.pnm.gov.my/xmlui/handle/123456789/1631), communication très proche du chapitre du présent ouvrage relatif aux lettres de Francis Light. 
de Merong Mahawangsa) et Taj al-Salatin (La couronne des rois) qui, comme l'accord de 1791, sont conservées à Londres.

Si les auteurs ou les copistes d'œuvres malaises identifiés comme originaires de Penang sont rares, il en existe tout de même un certain nombre. Dans la troisième partie, Jelani Harun nous en donne plusieurs exemples.

Il commence par Lebai ${ }^{14}$ Mamat, sur lequel on a très peu d'informations. On sait qu'il était illustrateur de pages de manuscrits et qu'à Penang, il a écrit Syair ${ }^{15}$ Yusuf (Poème de Joseph), daté du 24 septembre 1859, manuscrit conservé à la bibliothèque de l'université de Leyde, aux Pays-Bas. Il a rédigé cette œuvre d'après une histoire qu'il aurait entendue, ayant pour héros le prophète Joseph. Par rapport aux œuvres composées ou traduites en malais le concernant, Syair Yusuf sort de l'ordinaire. Il raconte, en effet, que la passion que ressentait pour lui Puteri Zulaikha (ou Zuliha) a suscité des calomnies qui l'ont presque conduit en prison.

L'auteur mentionne ensuite Lebai Che' Duh, un Jawi Peranakan de Penang, actif comme auteur et copiste vers 1889-1890. Il est à l'origine de trois manuscrits conservés à la bibliothèque de l'université de Leyde, obtenus de Snouck Hurgronje en 1936 : Hikayat Raja Budiman (Histoire du roi sage) et Surat al'-Anbiya (Les prophètes), en tant que copiste, et Kitab Fa'al (Livre des médicaments), semble-til, en tant qu'auteur. Ce dernier manuscrit, débute par une section (la plus longue) consacrée notamment aux maladies ordinaires, à celles causées par des esprits, aux rapports entre époux, à la façon de séduire une femme, laissant à penser que Lebai Che' Duh serait un sorcier/guérisseur (bomoh) connu de Penang. Vient ensuite une histoire sur le burung pingai (oiseau aux plumes jaune clair), puis un syair louant la beauté et le caractère d'une femme idyllique.

Jelani Harun poursuit en traitant du copiste Ibrahim bin Hakim Long Fakir Kandu alias Ibrahim Kandu. Né à Kedah en 1780 (p. 136) ${ }^{16}$, il est le frère d'Ahmad Rijaluddin bin Hakim Long Fakir Kandu ${ }^{17}$, qui a écrit Hikayat Perintah Negeri Benggala (1811), racontant son voyage de Penang au Bengale (p. 131) ${ }^{18}$. Ibrahim Kandu était aussi traducteur et secrétaire de Raffles, pour qui il a fait de nombreuses copies de manuscrits. Lui ayant également enseigné le malais, il est aussi connu sous le nom de Munshi Ibrahim. Il lui aurait même présenté Abdullah Munshi, alors encore jeune. Ibrahim Kandu effectue en particulier des copies pour Raffles, en 1810, d'undangundang (lois), comme Undang-Undang Laut Melaka (Lois maritimes de Malacca), conservées à la Royal Asiatic Society de Londres, faisant partie des plus anciennes

14. Titre pour une personne très croyante ou connaissant bien la religion musulmane.

15. Quatrains à monorimes.

16. Selon C. Skinner, dans «The author of the Hikayat Perintah Negeri Benggala » (Bijdragen tot de Taal-, Land- en Volkenkunde 132(2/3), 1976, (http://www.kitlv-journals.nl), on ne connaît pas exactement sa date de naissance puisqu'il dit, p. 201 : «Il est né à Kedah vers $1780 »$ (He was born in Kedah about 1780).

17. Kandu, présent dans leur nom, serait un mot tamoul, laissant penser que ce sont des Chulia, à savoir des «musulmans tamouls de la côte du Coromandel » (Tamil Muslims from the Coromandel coast) (C. Skinner, 1976, ibid., p. 196).

18. En réalité, ce texte n'est pas le récit de ce voyage, car presqu'entièrement consacré à « une description de Calcutta et de ses agglomérations adjacentes en $1810 »$ (a description of Calcutta and the adjoining settlements in the year 1810) (C. Skinner, 1976, ibid., p. 195). 
copies de manuscrits de Penang. On trouve aussi à la Bibliothèque nationale de Paris, des copies, réalisées par Édouard Dulaurier, de certains de ces manuscrits de Londres.

L'auteur termine cette troisième partie en se penchant sur le copiste Muhammad Naina Marikan (ca. 1904-1990), un Jawi Peranakan, qui, comme d'autres, avait transformé sa maison en scriptorium. Il possédait aussi une importante collection de manuscrits et de livres qui furent donnés à des bibliothèques ou à des particuliers, ou achetés par eux. Les trois copies qu'il a réalisées, dont parle Jelani Harun, datent de 1950 (celle de Hikayat Raja Budiman), de 1961 (celle de Hikayat Bayan Budiman " Histoire du perroquet sage ») et de 1963 (celle de Hikayat Anak Dara Quraisy « Histoire de la jeune fille Quraysh »), une époque plus tardive que la grande majorité des textes malais étudiés dans cet ouvrage. Ces copies se trouvent actuellement à l’Universiti Malaya, à Kuala Lumpur.

La quatrième partie de l'ouvrage porte sur l'histoire de l'imprimerie à Penang, l'une des premières régions de Malaisie où elle a été introduite, et où elle a prospéré avec vivacité au moins jusqu'à l'indépendance de 1957. Son histoire commence en 1806 et elle se développe rapidement grâce aux activités de la London Missionary Society. Elle connaît une nouvelle étape à partir de 1880 , car ce ne sont plus dès lors les imprimeries européennes qui dominent, mais les imprimeries chinoises, indiennes, Jawi Peranakan et malaises.

Cette activité foisonnante de l'imprimerie à Penang, répondant aux demandes d'ouvrages scolaires ainsi qu'aux demandes d'une société avide de modernité, attira journalistes, écrivains et éditeurs expérimentés du pays, mais aussi de Sumatra, comme Muhammad Ali bin Muhammad al-Rawi, propriétaire des United Press. On y imprimait non seulement des livres, mais aussi des journaux et des revues, dans lesquels paraissaient, entre autres, des nouvelles d'écrivains originaires de Penang, tels Muhammad Ariffin Ishak ou Muhammad Nur Ahmad. Pour Jelani Harun, tout ceci fit qu'au début du $\mathrm{XX}^{\mathrm{e}}$ siècle, l'île devint « le centre du développement de la langue et de la littérature » (pusat perkembangan bahasa dan sastera) (p. 159) (sous-entendu « malaise »), laissant ainsi supposer que d'autres centres n'existaient pas à la même époque dans le monde malais. Pourtant une intense activité linguistique et littéraire existait aussi ailleurs, en particulier à Singapour et à Riau. Jelani Harun explique que c'est également à Penang que les Kaum Muda (les Modernes), auxquels appartenaient des écrivains comme Syed Syeikh al-Hadi (1867-1934) et Ahmad Rashid Talu (18891939), introduisirent les idées progressistes de l'islam. Des revues œuvraient alors pour le progrès des Malais, comme Al-Ikhwan, fondée par Syed Syeikh al-Hadi, ou Suara Malaysia, publiée non pas en jawi, comme c'était généralement le cas à l'époque pour les publications en malais, mais en caractères latins (rumi). L'auteur insiste plus loin sur la présence du mot «Malaysia » dans son titre, ce qui est alors nouveau et rare.

Après cette analyse, sans transition, Jelani Harun fait une sorte de catalogue des imprimeries/maisons d'édition, donnant des exemples de leurs publications. Il commence par Criterion Press, fondée en 1883 par un Chinois d'Aceh, venu à Penang quand éclata la guerre entre Aceh et les Néerlandais. Cette maison d'édition publie, entre autres, à partir de $1894^{19}$, le journal en chinois Pinang Sin Poe, à partir de 1898,

19. Selon Lee Kuok Tiung et Mohd Safar Hasim (« Peranan Akhbar Cina dalam Artikulasi Isu-Isu Sejarah dan Pembentukan Negara-Bangsa » (Le rôle des journaux chinois dans l'arti- 
le journal en malais Chahyah Pulau Pinang (Lumière de Penang), en 1905, le livre de E. G. Cullin \& W. F. Zehnder, sur les débuts de l'histoire de Penang, The Early History of Penang (1592-1827), ainsi que de nombreuses hikayat ${ }^{20}$ malaises. Parmi les autres imprimeries/maisons d'édition, il cite Mercantile Press, qui publie, entre autres, la revue Malaya, le journal Idaran Zaman, le livre de Mohamad Yusof bin Sultan Maidin, Boria ${ }^{21}$ dan Benchananya (Le boria et ses dangers) (1922), Syair Boria (Le poème du boria) (1922) et le roman Iakah Salmah? (Est-ce bien elle Salmah ?) (1928) d'Ahmad Rashid Talu. Il cite aussi Jelutong Press que Syed Syeikh al-Hadi avait pu fonder en 1927, grâce au succès remporté par son célèbre roman Hikayat Faridah Hanom (Histoire de Faridah Hanom) (1925-1926) ${ }^{22}$. L'auteur livre également des informations sur Commercial Press, British-Malaya Press, Penang Premier Press, Al-Zainiyah Press, qui publie le journal Sahabat (L'ami), comptant Abdullah Hussain parmi ses auteurs ${ }^{23}$.

Après ce panorama des nombreuses imprimeries/maisons d'édition pionnières de Penang, l'auteur analyse plus en détail certains genres d'œuvres, en commençant par des pantun ${ }^{24}$ parus dans des journaux et des revues et écrits par des habitants de Penang, comme celui, nostalgique, qui regrette le temps d'avant Francis Light. C'est ensuite le tour de Pantun 481 (nombre représentant celui de ses strophes), dont l'original est conservé à la British Library de Londres. Son auteur est un certain Ahmar de Penang et il a été imprimé en jawi par United Press, également à Penang, en 1937.

Après avoir analysé des textes parus à l'occasion de la célébration de l'anniversaire des soixante ans (jubli intan) du gouvernement de la reine Victoria (par exemple déclarations (ucapan), louanges (puji-pujian) en jawi des habitants de Penang pour la reine, parues dans le Journal of the Straits Branch of the Royal Asiatic Society (JSBRAS) en 1886), l'auteur étudie tout particulièrement le Risalah Hayy bin Yaqzan (Histoire de Hayy bin Yaqzan), une traduction réalisée à partir de l'arabe par un écrivain de Penang, Ahmad Abdul Kadir bin Abdul Rahman, et publiée en jawi en 1918. Son auteur est Abu Bakr Muhammad bin Tufail al-Qaisi al-Andalusi, plus connu sous le nom de Ibn Tufail (1105-1185), écrivain, philosophe, médecin et notable de Grenade, en Espagne. Le titre de l'original est, semble-t-il, Hayy ibn Yaqdhan (XII ${ }^{\mathrm{e}}$ siècle), du nom du héros, titre que Jelani Harun n'indique pas expressément.

Jelani Harun explique que le Risalah Hayy bin Yaqzan est différent des œuvres arabes traduites en malais, car il est en quelque sorte un roman se penchant sur « la recherche par les humains de la réalité de leur présence dans ce monde » (pencarian

culation des questions historiques et dans la formation de l'État-nation), Jurnal Komunikasi, Malaysian Journal of Communication, Vol. 31 (1), 2015, p. 261, www.ukm.my/jkom/journal/ pdf_files/2015/V31_1_14.pdf), ce journal, appelé Penang Sin Poe (et non pas Pinang Sin Poe), est paru de 1895 (non pas 1894) à 1936.

20. Une hikayat est une longue histoire en prose.

21. Le boria est une forme théâtrale très populaire parmi les Malais de Penang, comprenant des scènes comiques, des chants, des danses, accompagnés de musique.

22. Jusqu'à présent, on ne sait pas s'il s'agit d'un original. Les personnages, les lieux, le contexte étant égyptiens, on pense qu'il pourrait s'agir d'une traduction ou d'une adaptation d'un roman égyptien, dont on n'a pas retrouvé la trace.

23. Après l'indépendance de la Malaisie, il deviendra l'un des Sasterawan Negara (Écrivain national), la plus haute distinction littéraire malaisienne.

24. Quatrains à rimes alternées. 
manusia tentang hakikat kewujudannya di dunia ini) (p. 220), que cette œuvre célèbre de la littérature arabe, traite aussi de la «philosophie du dévouement des humains à Dieu Le plus Pur et Le plus Haut » (falsafah keabdian manusia kepada Allah SWT) (p. 218). Il ajoute que grâce à sa traduction anglaise par Simon Ockley ${ }^{25}$, parue en 1708 , il s'est rapidement diffusé en Europe ${ }^{26}$. Il ne dit pas cependant à partir de quelle langue elle a été réalisée. Celle-ci est en fait la première traduction anglaise réalisée à partir de l'original arabe et s'intitule The improvement of human reason exhibited in the life of Hai ebn Yokdhan ${ }^{27}$. Jelani Harun explique qu'entre le XIV et le XIX ${ }^{\mathrm{e}}$ siècles, cette œuvre a été traduite non seulement en anglais, mais aussi en néerlandais, en allemand, en français et en espagnol, signe de son succès au niveau international, et qu'elle a même inspiré Daniel Defoe pour écrire Robinson Crusoe (cf. p. 223) ${ }^{28}$. Signalons que pendant cette période, elle a été aussi traduite en hébreu (la première, réalisée au XIV siècle) ${ }^{29}$, sans doute celle dont veut parler Jelani Harun quand il indique ce siècle, et en latin. Pour ce qui est de l'espagnol, elle n'aurait pas été traduite dans cette langue au XIX $\mathrm{X}^{\mathrm{e}}$ siècle, mais pour la première fois en $1900^{30}$.

L'auteur achève cette quatrième partie en examinant le rôle important des enseignants dans le développement de la langue et de la littérature malaise à Penang, à travers la revue Panduan Guru (Guide pour les enseignants), publiée dès 1922 par la Persekutuan Guru-Guru Melayu Pulau Pinang (Association des Enseignants malais de Penang), fondée en 1920. Elle publiait beaucoup de choses concernant la profession d'enseignant, mais aussi des nouvelles, des histoires drôles, des poèmes et des articles sur la défense de la langue et de la littérature. La revue cessa de paraître en 1925 et fut remplacée par Majalah Guru (Revue des enseignants).

La cinquième et dernière partie est consacrée aux œuvres produites dans deux régions de Penang : Balik Pulau et Seberang Perai.

Balik Pulau, dont le nom est peut-être lié à sa position derrière les collines le séparant de George Town, fut l'un des premiers lieux d'installation de Malais à Penang.

25. « Ockley » et non pas « Oakley » comme indiqué dans l'ouvrage de Jelani Harun.

26. Il est même devenu un " best-seller influent à travers l'Europe de l'Ouest aux XVII ${ }^{e}$ et XVIII ${ }^{\mathrm{e}}$ siècles " (infuential best-seller through-out Western Europe in the 17th and 18th century) (Fredrick Kennard, Thought Experiments: Popular Thought Experiments in Philosophy, Physics, Ethics, Computer, Science \& Mathematics, Lulu.com, 2015, p. 150). https://books.google.fr/books?id=sX-pCQAAQBAJ\&printsec $=$ frontcover\&dq=Fredrick + Kennard,+ Thought +Experiments\&hl $=$ fr\&sa $=X \&$ redir esc $=y \# v=$ onepage $\& \mathrm{q}=$ Fredrick $\% 20$ Kennard $\% 2 \mathrm{C} \% 20$ Thought $\% 20$ Experiments $\& \mathrm{f}=$ falsep).

27. Hayy ben Yaqdhân, Roman philosophique d'Ibn Thofaïl, Texte arabe avec les variantes des manuscrits et de plusieurs éditions et traduction française, $2^{\mathrm{e}}$ édition, revue, augmentée et complètement remaniée par Léon Gauthier, Beyrouth, Imprimerie Catholique, 1936, p. XXIX-XXX (https://iqbal.hypotheses.org/1372). À noter que la première traduction anglaise de l'œuvre date de 1674. Elle a été réalisée par Georges Keith à partir de la traduction latine d'Edward Pococke de 1671 (ibid., p. XXIX).

28. Jelani Harun donne cette information en se basant sur l'ouvrage intitule The journey of the soul: the story of Hai bin Yaqzan, as told by Abu Bakr Muhammad bin Tufail (Londres, Octagon, 1982), qui est une traduction anglaise du Hayy ibn Yaqdhan, réalisée par Riad Kocache.

29. « La traduction hébraïque d'auteur inconnu, dont Moïse de Narbonne a donné, en 1349, un commentaire en hébreu » (Hayy ben Yaqdhân, Roman philosophique d'Ibn Thofaïl, 1936, op. cit., p. XXIX).

30. Ibid., p. XXXII. 
L'auteur n'en précise pas l'époque. Il dit cependant, plus loin (p. 246) qu'il existait des « implantations » (kawasan-kawasan) à Balik Pulau remontant à une époque aussi lointaine que celle de la légende de Nakhoda Ragam, dont on a parlé plus haut. Il explique aussi qu'une tradition intellectuelle, surtout centrée sur l'enseignement de la religion, s'y est développée.

On a peu d'informations sur la présence de manuscrits à Balik Pulau. On sait que Hikayat Raja Budiman y a été copié par Dolah (copie conservée à la Royal Asiatic Society). Une copie du Sabil Al-Muhtadin (Le chemin des gens correctement guidés), écrit en 1779 par Syeikh Muhammad Arshad bin Abdullah al-Banjari (1710-1812), sur la base du Sirat al-Mustakim (Le droit chemin), de Syeikh Nuruddin Al-Raniri de 1634, se trouve dans la collection privée de Haji Mustapa bin Kassim et de Rahilah binti Hamzah. Le nom du copiste et du lieu de production n'y sont pas mentionnés. Il est cependant probable que la copie ait été réalisée à Balik Pulau pour les besoins de l'enseignement de la religion dans les écoles coraniques. Ces mêmes personnes possèdent un manuscrit intitulé Mir'at al-Asrar ${ }^{31}$, établi par Syeikh Sirajuddin bin Syeikh Jalaluddin à Aceh, recopié par un certain Haji Ismail bin Lebai Ibrahim à Kampung Sungai Rusa (Balik Pulau), en 1887.

A côté de cette tradition écrite subsiste à Balik Pulau une tradition orale (mythes d'origine par exemple), perpétuée par des personnes âgées.

Autrefois connue sous le nom de Province Wellesley, Seberang Perai, en face de l'île sur la péninsule, a été cédée en 1800 par le sultan de Kedah à la Compagnie anglaise des Indes orientales. Pour en retracer l'histoire ancienne, vu le manque de sources écrites, les historiens ont recours, par exemple, à la tradition orale et à l'archéologie. C'est là que se situerait l'origine de l'enseignement islamique à Penang et, aujourd'hui encore, on y trouve un grand nombre d'écoles coraniques ayant modernisé leur enseignement. Cette région a donné naissance à des spécialistes aussi bien traditionalistes que progressistes. Certains enseignants d'écoles coraniques étaient aussi des écrivains, des copistes, produisant des ouvrages destinés non seulement aux étudiants mais aussi à un public plus large. L'art islamique s'y est développé sous la forme de Hadrah et Maulud Jawi.

Les Hadrah sont des chants didactiques, accompagnés par des tambourins (à une face) (rebana). Les groupes de Hadrah donnent des représentations à l'occasion de fêtes et de mariages. Ces textes n'ayant jamais été mis par écrit, ils n'existent que sous forme orale mémorisée par les chanteurs.

Les Maulud Jawi sont des chants à la gloire du prophète Mahomet, interprétés par des femmes à l'occasion de la célébration de la fête de Sa naissance. Ces chants ont été mis par écrit par Imam Mat Saman bin Lebai Kadin, sous le titre de Kitab Maulud Jawi. Les représentations de Maulud Jawi se déroulent chez des particuliers. Seules les femmes y assistent, alors qu'à l'extérieur les hommes aident à la préparation des

\footnotetext{
31. Il s'agit sans doute du Mir'at al-Asrar de 'Abd al-Rahman Chishti (cf. Richard M. Eaton, Munis D. Faruqui, David Gilmartin, Sunil Kumar, éds., Expanding Frontiers in South Asian and World History: Essays in Honour of John F. Richards, Cambridge University Press, 2013, p. 131) (https://books.google.fr/books?id=h0_xhdCScQkC\&pg=PA131\&lp$\mathrm{g}=\mathrm{PA} 131 \& \mathrm{dq}=\mathrm{Mir} \% \mathrm{E} 2 \% 80 \% 99 \mathrm{at}+\mathrm{al}-\mathrm{Asrar}++\mathrm{de}+\% \mathrm{E} 2 \% 80 \% 9 \overline{8} \mathrm{Abd}+\mathrm{al}-\mathrm{Rahman}+\mathrm{Chishti} \&-$ source=bl\&ots=1x-HeM16SM\&sig=CG-hRbIAVz09H66JOU9zqfK0Qko\&hl=fr\&sa=X\&ved=0ahUKEwiJ4IjRiKrVAhXFMhoKHeU9BW4Q6\#v=onepage\&q=Mir\%E2\%80\%99at\%20al-Asrar\%20 $\% 20 \mathrm{de} \% 20 \% \mathrm{E} 2 \% 80 \% 98 \mathrm{Abd} \% 20 \mathrm{al}-$ Rahman $\% 20$ Chishti\&f=false).
} 
mets destinés au repas cérémoniel. Le Kitab Maulud Jawi ressemble au Kitab Kanz al-'Ula, écrit par Sayid Muhammad Zain al'Abidin al-'Idrus (1795-1878), plus connu sous le nom de Tok Ku Tuan Besar de Terengganu, et il est très probable qu'il en soit une copie.

Jelani Harun a fait un travail de recherche minutieux, tentant de détecter, dans la mesure du possible, les textes produits à Penang. Une tâche peu aisée, surtout en ce qui concerne les manuscrits qui, bien souvent, ne comportent ni nom d'auteur ou de copiste, ni date, ni lieu de production et dont la grande majorité des textes originaux se trouve à l'étranger. Il a cependant réussi à en trouver un assez grand nombre, en faisant des recherches bibliographiques, en allant les consulter en Malaisie, mais aussi à l'étranger, en les cherchant également chez des particuliers, leurs héritiers, ou des personnes les ayant connus, certains n'étant cependant pas très coopératifs ( $p .148$ : « mon expérience lors de mes recherches sur Muhammad Naina Marikan a été assez éprouvante et fatigante à cause de l'attitude d'héritiers et de certaines personnes ne voulant pas coopérer comme il le faudrait $»^{32}$ ).

Cet ouvrage est une contribution importante à l'histoire des débuts de la littérature malaise ancienne et moderne. Il apporte également des informations sur les origines de l'histoire de Penang. Les nombreuses illustrations et les extraits de textes ou, plus rarement, des textes complets, rendent de même plus vivantes les analyses. On peut cependant regretter quelquefois l'aspect inventaire de l'ouvrage, notamment lorsque l'auteur fournit la liste commentée des imprimeries/maisons d'édition de Penang. Il est vrai qu'il pourrait aussi être utilisé comme une sorte de catalogue des textes (manuscrits, imprimés et oraux) en malais produits à Penang depuis ses origines jusqu'au début du $\mathrm{XX}^{\mathrm{e}}$ siècle.

Cependant, cet ouvrage est beaucoup plus qu'un catalogue, car il comprend, la plupart du temps, des analyses précises et détaillées. Certains passages pourtant manquent de rigueur et donnent une impression de flou, laissant le lecteur dans le doute (par exemple, quand l'auteur parle d'accords entre le sultan de Kedah et la Compagnie anglaise des Indes orientales, signés en 1771, ou des traductions du Hayy ibn Yaqdhan).

Le travail de Jelani Harun nous semble novateur. Nous ne connaissons pas, en effet, d'ouvrage traitant de l'histoire d'un centre de production littéraire en malais à la fois sous l'angle de la tradition orale, des textes (manuscrits et imprimés), des auteurs ou des copistes, ainsi que des imprimeries et des maisons d'édition. Sa démarche ressemble à celle de G.W.J. Drewes que signale Henri Chambert-Loir dans son article « La littérature malaise ancienne $»^{33}:$ « En annexe à un ouvrage consacré à un

\footnotetext{
32. "pengalaman penulis dalam membuat kajian terhadap Muhammad Naina Marikan agak mencabar dan meletihkan, lantaran sikap sesetengah waris dan orang tertentu yang enggan memberikan kerjasama yang diperlukan ».
}

33. In Henri Chambert-Loir, éd., La littérature indonésienne. Une introduction, Cahier d'Archipel 22, Collection Jeanne Cuisinier. INALCO, 1994, p. 9-41. 
traité religieux arabe et à ses adaptations indonésiennes, G.W.J. Drewes a tenté une expérience sans précédent, celle de repérer dans les collections de manuscrits malais tous ceux provenant de Palembang $»^{34}$ (p. 24). C'est bien ce qu'a tenté de faire Jelani Harun dans le cas de Penang, mais pas uniquement pour ce qui est des manuscrits malais.

MONIQUe ZAINI-LAJOUBERT

Helen M. Creese, Bali in the Early Nineteenth Century: The Ethnographic Accounts of Pierre Dubois, Verhandelingen van het Koninklijk Instituut voor Taal-, Land- en Volkenkunde 305, Brill, Leiden \& Boston, 2016, XIX, 826 p. (26 ill.), cartes, fac. sim. ISBN : 978-90-04-31582-2 ; 90-04-31582-9

Dans cet imposant volume, Helen Creese analyse d'une façon extrêmement minutieuse les modalités de la rencontre entre Néerlandais et Balinais à partir des écrits de Pierre Dubois, qui servit à Bali comme administrateur civil de mars 1828 à mai 1831. Sa mission officielle était de recruter des soldats pour servir dans les troupes coloniales combattant à Java l'insurrection du prince Diponegoro. En dehors des rapports administratifs qu'il devait adresser périodiquement à sa hiérarchie, le comité de direction de la Société batave des arts et des sciences (Bataviaasch Genootschap van Kunsten en Wetenschappen) lui demanda en mai 1829 de rendre compte de ses expériences personnelles à Bali. Il a travaillé à ce projet, demeuré inachevé, jusqu'à son décès en 1838, en rédigeant une série de lettres en français, réunies sous l'intitulé Légère Idée de Balie en 1830. Témoignage oculaire original de la vie balinaise dû à un Européen établi dans l'île, ces textes peuvent être considérés comme le premier compte rendu ethnographique de Bali.

Philologue de formation, Helen Creese avait initialement conçu son étude comme un simple travail d'édition et de traduction des lettres de Dubois. Au fil des ans, elle a élargi son propos de façon à y intégrer une histoire des relations entre Néerlandais et Balinais depuis l'ère napoléonienne jusqu'à la première expédition militaire contre Bali en 1846. Si bien que son livre se compose de trois parties : l'édition originale de Légère Idée de Balie en 1830 et sa traduction annotée en anglais y sont précédées d'une présentation circonstanciée du contexte historique et culturel du séjour de Dubois à Bali qui occupe plus de 300 pages.

Pierre Dubois est né en 1781 à Mouscron, un village wallon dans la province de Hainaut. Après avoir servi comme officier dans la Grande Armée, et

34. L'ouvrage de Drewes est Directions for Travellers on the Mystic Path, La Haye, Nijhoff, 1977 (VKI 81). L'annexe se trouve p. 198-244. 